\title{
COMMENTS
}

Comments are short papers which criticize or correct papers of other authors previously published in the Physical Review. Each Comment should state clearly to which paper it refers and must be accompanied by a brief abstract. The same publication schedule as for regular articles is followed, and page proofs are sent to authors.

\section{Comment on "On the hidden supersymmetry in stochastic quantization"}

\author{
E. Gozzi \\ Department of Theoretical Physics, University of Trieste, Strada Costiera 11, P.O. Box 586, Trieste, 34136, Italy
}

(Received 28 December 1989)

\begin{abstract}
There has recently been some criticism by Noga on the hidden supersymmetry discovered by Parisi and Sourlas (and many other authors) in parabolic stochastic equations. We will show in this paper how all points raised by Noga against this supersymmetry are due to a misreading of the existing literature.
\end{abstract}

\section{INTRODUCTION}

It has been ten years since Parisi and Sourlas [1] discovered that stochastic-correlation functions built out of solutions of elliptic stochastic equations could also be obtained as correlation functions from a sort [in this case, rather than a true supersymmetry (translation in superspace) it is a superrotation in superspace] of supersymmetric generating functional. Later on various authors [2] realized also that parabolic stochastic equations have solutions whose correlation functions can be derived from a supersymmetric generating functional (this supersymmetry is a true one in the sense of a translation in superspace). (The relation between supersymmetry and stochastic equations is also known as Nicolai mapping [3] and it was discovered independently of stochastic processes and used to give various important applications in supersymmetric models.)

Recently Noga [4] sharply criticized this supersymmetry. Noga wanted to prove, in his paper, that this supersymmetry is a fake phenomenon and is only due to (quoting from the paper of Noga [4]) "formal manipulations" that lead to "incorrect results and proliferation of mistakes." In particular he finds three major contradictions to which the supersymmetry leads.

In the next three sections we will analyze in detail these three contradictions and see that they stem from having misread the relevant literature [2].

\section{FIRST CONTRADICTION}

Let us follow Noga. He says if the supersymmetry is there only with periodic boundary conditions [2] (BC's) then these BC's lead to the following contradiction [4]: take the expectation value of an observable $E(\phi)$. They are given by

$$
\langle E(\phi)\rangle=\int d^{n} \phi E(\phi) \widetilde{P}\left(\phi, t \mid \phi_{0}, t_{0}\right)
$$

For a stochastic process without a drift force the transition probability is given by

$$
\widetilde{\boldsymbol{P}}\left(\phi, t \mid \phi_{0}, t_{0}\right)=\frac{1}{\left(t-t_{0}\right)^{n / 2}} \exp \left[-\frac{\left(\phi-\phi_{0}\right)^{2}}{t-t_{0}}\right) .
$$

Let us now choose, as observable $E(\phi)$, just the constant $E(\phi)=1$; then, because of the periodic BC in $\widetilde{P}$, we obtain, from (2.1),

$$
1=\int d^{n} \phi\left[\frac{1}{\left(t-t_{0}\right)^{n / 2}}\right] .
$$

In the limit of $t \rightarrow \infty$ this gives

$$
\begin{aligned}
& 1=0 \int d^{n} \phi, \\
& 1=0,
\end{aligned}
$$

and, as Noga says [4], this is a contradiction.

In the last paper of Ref. [2] we repeatedly stressed that we use periodic BC's only in the generating functional $Z_{\mathrm{SS}}^{\mathrm{FP}}[J]$ (we refer the reader to the last paper of Ref. [2] for the notation, FP is for Fokker-Planck), and only between the configurations at 0 and at $\tau$, and not at every $t$ as Noga says. This means

$$
Z_{S S}^{F P}[J]=\int \mathscr{D} \phi P(\phi(0)) e^{S(\phi(0)) / 2} \mathscr{D}(\phi(2 \tau)) e^{-S(\phi(2 \tau)) / 2} \widetilde{D}^{\prime \prime} \phi \exp \left(\int_{0}^{2 \tau} \mathcal{L}_{\mathrm{SS}}^{\mathrm{FP}} d \tau^{\prime}-\int_{0}^{2 \tau} J \phi d \tau^{\prime}\right)
$$


The correlation functions are given by

$\left\langle\phi\left(\tau_{1}\right) \phi\left(\tau_{2}\right) \cdots \phi\left(\tau_{l}\right)\right\rangle=\left.\frac{\partial^{l} Z_{\mathrm{SS}}^{\mathrm{FP}}[j]}{\partial J\left(\tau_{1}\right) \cdots \partial J\left(\tau_{l}\right)}\right|_{J=0}$

where $0<\tau_{1}<\tau_{2}<\cdots<\tau_{l}<2 \tau$. The configurations that are equal are only those at the times 0 and $2 \tau$ and not at every instant $\tau_{i}$ as Noga says. Note that $Z_{\mathrm{SS}}^{\mathrm{FP}}[J]$ is not the Witten index [5] even if it is built with a periodic BC, because it has inserted an external current. The reason we use this generating functional, and are not bothered too much by the BC, is because in the limit of $\tau \rightarrow \infty$ this supersymmetric generating functional goes into the standard [2] $Z^{\mathrm{FP}}$, and, being built out of a Markov process, it will lose its memory of the initial conditions.

\section{SECOND CONTRADICTION}

The second contradiction that Noga found is the following: because of the supersymmetry, the transition probability satisfies the standard composition law of Chapman and Kolmogoroff. Noga used the "det" that was calculated in Ref. [2] and wrote down

$\widetilde{P}\left(\phi, t \mid \phi_{0}, t_{0}\right)=\int_{\phi_{0}, t_{0}}^{\phi, t} \widetilde{D}^{\prime \prime} \phi\left(\sinh \int \frac{1}{2} \frac{\partial F}{\partial \phi}\right) \exp \left[\int \frac{(\dot{\phi}+F)^{2}}{4}\right]$

Because of the "sinh" this probability will not satisfy the standard Chapman-Kolmogoroff formula

$\widetilde{P}\left(\phi, t \mid \phi_{0}, t_{0}\right)=\int \widetilde{P}\left(\phi, t \mid \phi_{1}, t_{1}\right) \widetilde{P}\left(\phi_{1}, t_{1} \mid \phi_{0}, t_{0}\right) d \phi_{1}$.

Well, we never wrote down the formula (3.1). Following Ref. [2] closely everyone will realize that the operational formalism associated to (2.4) is a two-component formalism, as is explained in detail in formula (38) of the last paper of Ref. [2]. (This two-component formalism appeared first in the paper of Witten [5], and it was further studied by Van Holten [3].) That means that the probability is also a two-component vector

$$
\widetilde{P}=\left(\begin{array}{l}
\widetilde{P}_{\text {forw }} \\
\widetilde{P}_{\text {back }}
\end{array}\right)
$$

and it evolves according to the following equation:

$$
\left[\begin{array}{c}
\widetilde{P}_{\text {forw }}(t) \\
\widetilde{P}_{\text {back }}(t)
\end{array}\right)=\left(\begin{array}{cc}
\exp \left(-\hat{H}_{\text {forw }}^{\mathrm{FP}} t\right) & 0 \\
0 & \exp \left(-\hat{H}_{\text {back }}^{\mathrm{FP}} t\right)
\end{array}\right)\left(\begin{array}{c}
\widetilde{P}_{\text {forw }}(0) \\
\widetilde{P}_{\text {back }}(0)
\end{array}\right) .
$$

Each component of this "vector" $\widetilde{P}_{\text {forw/back }}$ probability has an evolution kernel $\exp \left(-\hat{H}_{\text {forw/back }}^{\mathrm{FH}}\right)$ which can be represented by a purely bosonic path integral that has a purely "exponential" form without any "sinh" and so each of the two components of $\widetilde{P}$ satisfies the ChapmanKolmogoroff relation

$$
\begin{aligned}
& \widetilde{P}_{\text {forw }}\left(\phi, t \mid \phi_{0}, t_{0}\right)=\int d \phi_{1} \widetilde{P}_{\text {forw }}\left(\phi, t \mid \phi_{1}, t_{1}\right) \widetilde{P}_{\text {forw }}\left(\phi_{1}, t_{1} \mid \phi_{0}, t_{0}\right), \\
& \widetilde{P}_{\text {backw }}\left(\phi, t \mid \phi_{0}, t_{0}\right) \\
& \quad=\int d \phi_{1} \widetilde{P}_{\text {backw }}\left(\phi, t \mid \phi_{1}, t_{1}\right) \widetilde{P}_{\text {backw }}\left(\phi_{1}, t_{1} \mid \phi_{0}, t_{0}\right) .
\end{aligned}
$$

So this solves the second contradiction. It also brings to light what is the real nature of this supersymmetry; it ties up forward and backward dynamics in a unique formalism. (In addition to the last of Ref. [2], this aspect of the FP dynamics has also been studied in great detail by Claudson and Halpern [2] who stressed repeatedly the presence of two stochastic processes in the supersymmetric formulation.) The relations that we [2] have derived from this supersymmetry (such as Ward identities and the like) have always been checked against the standard forward dynamics; this means we always looked at what the Ward identities become in the limit of $\tau \rightarrow \infty$. We found that they were reproducing standard relations valid for the forward dynamics (such as, for example, the fluctuation-dissipation theorem [2]) and there was never any contradiction between supersymmetric dynamics (in the limit $\tau \rightarrow \infty$ ) and standard forward dynamics. Of course, as we stressed in the last of Ref. [2], this formalism works if there is an appropriate drift force that leads to a normalizable forward distribution. In the case, for example, of constant drift force $\partial F / \partial \phi=0$, the equilibrium distributions are not normalizable and so the correspondence $Z_{\text {SS }}^{\text {FP }} \rightarrow Z_{\text {forw }}^{F P}$ should not be used, just because the forward distribution itself does not exist. Another case to point out is that of $F=0$ : here the two components of Eq. (3.3) are completely equivalent and decoupled, and we can choose any one of them. The sypersymmetry in this case is empty because there are no ghosts, but it is not contradictory: we just get a doubling of the standard formalism.

In any case, if we had found some differences between the forward formalism and the supersymmetric one, then we would agree with Noga that the supersymmetry is due to our mistakes, but we never found any of these contradictions. On the contrary the supersymmetry helped to derive standard relations in a more elegant manner and bring to light the beautiful Onsager principle of microreversibility as was independently discovered by Feigelman and Tsvelik [2].

\section{THIRD CONTRADICTION}

In this third contradiction, Noga again insists on the famous "det" formula coming from supersymmetry.

The determinant somehow relates the noise $\eta$ [2] with the solution $\phi$ of the Langevin equation. He shows that when we have no drift force the "det" is zero (if we insist with the supersymmetry); this means that given an $\boldsymbol{\eta}$ there is no $\phi$ associated and that is absurd, and so we have to abandon the supersymmetric manner to calculate the "det." Let us remember that the "det" [2] is

$$
J[\phi]=k \sinh \int \frac{\partial F}{\partial \phi} .
$$


Noga says if $F$ is zero (or constant) we get $J[\phi]=0$. Here the mistake of Noga again is that he does formal manipulations without reading properly the papers he criticizes [2]. If he had read them, he would have found (formula (13) of the last paper of Ref. [2]) that the formula for the "det" is actually

$$
J[\phi]=\frac{\sinh \int \partial F / \partial \phi}{\operatorname{Det} \partial_{t}}=\frac{\operatorname{Det}\left(\partial_{t}+\partial F / \partial \phi\right)}{\operatorname{Det} \partial_{t}} .
$$

Now for $F=0$ we would get, from (4.2),

$$
J[\phi]=\frac{\operatorname{Det}_{t}}{\operatorname{Det} \partial_{t}}=1
$$

and this proves that, given a noise $\eta$, even in the free case we always have a solution $\phi$, as one expects also from a nonsupersymmetric analysis. This confirms that the supersymmetry is not in contradiction with the usual analysis but confirms it.

\section{ACKNOWLEDGMENTS}

I thank P. Damgaard, S. Marculescu, K. Okano, M. Reuter, and L. Schuelke for bringing the paper of Noga to my attention and for discussions. I also thank Prof. M. Halpern for reminding me that also his paper [2] is totally based on the presence of two stochastic processes in the supersymmetric formulation.
[1] G. Parisi and N. Sourlas, Phys. Rev. Lett. 43, 744 (1979); B. McClain et al., Ann. Phys. (N.Y.) 140, 232 (1982).

[2] M. V. Feigelman and A. M. Tsvelik, Zh. Eksp. Teor. Fiz. 83, 1430 (1982) [Sov. Phys. JETP 56, 823 (1982)]; G. Parisi and N. Sourlas, Nucl. Phys. B206, 321 (1982); S. Cecotti and L. Girardello, Ann. Phys. (N.Y.) 145, 81 (1983); C. Bender et al., Nucl. Phys. B219, 61 (1983); F. Cooper and B. Freedman, Ann. Phys. (N.Y.) 146, 262 (1983); H. Nakazato et al., Prog. Theor. Phys. 70, 298 (1983); P. H. Damgaard and K. Tsokos, Lett. Math. Phys. 8, 535 (1984); S. Chaturvedi et al., Z. Phys. B 57, 249 (1984); R. Graham et al., Phys. Lett. 109A, 436 (1985); M. Claudson and M. B. Halpern, Ann. Phys. (N.Y.) 166, 33 (1986); G. JonaLasinio, in Quantum Field Theory, Proceedings of the International Symposium, Positano, Italy, 1985, edited by F.
Mancini (North-Holland, Amsterdam, 1986); J. ZinnJustin, Nucl. Phys. B275, 135 (1986); E. Gozzi, Phys. Rev. D 28, 1922 (1983); 30, 1218 (1984).

[3] H. Nicolai, Phys. Lett. 89B, 341 (1980); Nucl. Phys. B176, 419 (1980); Phys. Lett. 117B, 408 (1982); J. W. Van Holten, in Unification of the Fundamental Particle Interactions II, Proceedings of the Europhysics Study Conference, Erice, Italy, 1981, edited by J. Ellis and S. Ferrara, Ettore Majorana International Science Series: Physical Sciences, Vol. 15 (Plenum, New York, 1983), p. 505 (QCD 161:E85:1981); V. De Alfaro et al., Nucl. Phys. B255, 1 (1985); K. Dietz et al., ibid. B255, 149 (1985).

[4] M. Noga, Phys. Rev. D 38, 3158 (1988).

[5] E. Witten, Nucl. Phys. B188, 513 (1981); S. Cecotti and L. Girardello, Phys. Lett. 110B, 39 (1982). 